\title{
ADESÃO ÀS MEDIDAS DE MITIGAÇÃO DA COVID-19 NO MUNICÍPIO DE SANTA CRUZ DO SUL-RS
}

\author{
VARGAS, G.R.1-; MOYSÉS, M.P.P.2-; CARNEIRO, M.3-;RENNER, J.4-; KOEPP, J.5-; GAEDKE, M. A.6; PAZ, I7; \\ SCHNEIDER, A.P.H.8;
}

PALAVRAS-CHAVE: : COVID-19. ISOLAMENTO SOCIAL. PANDEMIA.

\begin{abstract}
RESUMO
Avaliar a adesão ao uso de máscara e às medidas de distanciamento social no município de Santa Cruz do SulRS. Recorte de estudo transversal de base populacional na região do Consórcio Intermunicipal de Serviços do Vale do Rio Pardo (CISVALE), compreendendo 14 municípios do Vale do Rio Pardo, região central do Rio Grande do Sul. Foram realizadas 3 etapas de coleta de dados quinzenais. Ocorreram amostragens sistemáticas dos setores censitários, sorteio aleatório dos domicílios, sorteio de uma pessoa em cada domicílio, realizando exame de teste de diagnóstico rápido para a detecção de anticorpos IgG e lgM. Para a coleta de dados foi utilizado um formulário on-line, sendo analisados por meio de estatística descritiva. Amostra composta de um total de 1158 indivíduos entrevistados. A média da idade foi de 49,5 ( \pm 19$)$ anos, $62,7 \%$ eram do sexo feminino e $80,4 \%$ residiam na zona urbana. Em relação ao distanciamento social, a maioria dos entrevistados $(55,71 \%)$ relatou que seguem a medida parcialmente. Em relação ao uso de máscara ao sair de casa, 95,66\% relatam que usam máscara ao sair. É notório que a implementação do distanciamento e o uso de máscaras é um grande desafio na realidade do nosso país. Essa estratégia é de extrema importância para evitar o colapso dos serviços de saúde e redução do número de mortes, de forma que uma adesão efetiva garante a proteção das comunidades da região.
\end{abstract}

\section{ADHESION TO COVID-19 MITIGATION MEASURES IN THE MUNICIPALITY OF SANTA CRUZ DO SUL}

\author{
KEYWORDS: COVID-19. SOCIAL ISOLATION. PANDEMIC.
}

\begin{abstract}
To evaluate adherence to the use of a mask and to measures of social distance in the municipality of Santa Cruz do Sul. Cross-sectional population-based study that measured the seroprevalence of SARS-CoV-2 in the CISVALE region, comprising 14 municipalities in the Vale from Rio Pardo, central region of Rio Grande do Sul. Three fortnightly data collection steps were performed. There were systematic sampling of the census sectors, random drawing of households, drawing of one person in each household, carrying out a rapid diagnostic test for the detection of IgG and IgM antibodies For data collection an online form was used, being analyzed using descriptive statistics. Sample composed of a total of 1158 individuals interviewed. The average age was 49.5 ( \pm 19 ) years, $\mathbf{6 2 . 7 \%}$ were female and $\mathbf{8 0 . 4 \%}$ lived in the urban area. Regarding social distance, the majority of respondents (55.71\%) reported that they more or less follow the measure. Regarding the use of a mask when leaving home, $95.66 \%$ report that they wear a mask when leaving. It is clear that the implementation of distance and the use of masks is a major challenge in the reality of our country. This strategy is extremely important for not filling health services and reducing the number of deaths, so that an effective adhesion guarantees the protection of the communities in the region.
\end{abstract}

\footnotetext{
1 Acadêmica do curso de Enfermagem na Universidade de Santa Cruz do Sul.

2 Acadêmica do Curso de Enfermagem na Universidade de Santa Cruz do Sul.

3 Doutor em Medicina a, e docente do Departamento de Ciências da Vida, Universidade de Santa Cruz do Sul

4 Doutora em Medicina e Ciências da Saúde, e docente do Departamento de Ciências da Vida, Universidade de Santa Cruz do Sul

5 Doutora em Medicina e Ciências da Saúde, e docente do Departamento de Ciências da Saúde, Universidade de Santa Cruz do Sul

6 Doutora em Saúde Coletiva, e docente do Departamento de Ciências da Saúde, Universidade de Santa Cruz do Sul

7 Mestre em Educação, e docente do Departamento de Ciências da Vida. Universidade de Santa Cruz do Sul

8 Orientadora Doutora em Saúde Coletiva, e docente do Departamento de Ciências da Vida, Universidade de Santa Cruz do Sul <anahelfer@unisc.br>
} 


\section{INTRODUÇÃO}

A humanidade tem enfrentado desde dezembro de 2019 a pandemia de COVID-19, pelo novo coronavírus (SARS-CoV-2) (AQUINO et al., 2020). Sua disseminação ocorreu de forma tão rápida que a Organização Mundial da Saúde (OMS) declarou que o mundo estava enfrentando uma pandemia no dia 11 de março de 2020. Essa crise sanitária global, originária da China, gerou uma emergência em saúde pública por seu nível de mortalidade e principalmente por sua alta taxa de transmissibilidade (AQUINO, et al., 2020). A doença consegue se disseminar através de gotículas contaminadas da região orofaríngea da pessoa infectada para a pessoa saudável, como por exemplo, por meio de espirro, saliva, tosse, catarro, e até mesmo pelo contato com superfícies contaminadas (BRASIL, 2020).

A transmissão da doença é alarmante pelo fato de que esse vírus possui um tempo de incubação médio (sendo esse o tempo de se infectar até início dos sintomas) de 5-6 dias (tolerando em até 12 dias) e por sua possibilidade de transmissão a partir de pessoas assintomáticas, pré-sintomáticas e com sintomas leves (AQUINO, et al., 2020).

Os sintomas mais comuns da doença são a tosse, a febre, a coriza, a dificuldade para respirar, dor de garganta, a perda de olfato, a alteração do paladar, os distúrbios gastrintestinais, a astenia (cansaço), a hiporexia (diminuição do apetite) e a dispneia (BRASIL, 2020).

Esses sintomas podem evoluir para um caso clínico mais grave com possível pneumonia, síndrome do desconforto respiratório agudo, arritmias, insuficiência cardíaca, falência múltipla de órgãos, choque séptico, entre outros que necessitam de uma atenção especializada e contínua por se encontrarem hemodinamicamente instáveis. (MEMON, et al., 2021).

Desde os primeiros registros na China em dezembro de 2019 até o dia 17 de abril de 2021 foram confirmados 141.057.106 casos de COVID-19 no mundo. Deste total, 3.015.043 evoluíram a óbito. Frente ao caos estabelecido pelo coronavírus foi preciso encontrar um meio para controlar a transmissibilidade para, assim, conseguir reduzir o número de casos, internações e mortes. Com isso, foram implementadas estratégias para a contenção da doença como distanciamento social e o uso de máscaras (MEMON, et al., 2021).

O distanciamento social se caracteriza pela diminuição de interação entre a população, sendo utilizado quando há preocupação de haver pessoas infectadas ainda não identificadas e circulando entre as demais, viabilizando o contágio. Com essa medida, associada ao uso de máscaras em locais públicos, é possível reduzir a transmissão do vírus e com isso evitar o colapso dos sistemas de saúde, diminuindo o número de mortes (WILDER et al., 2020). Esse estudo teve como objetivo avaliar a adesão ao uso de máscaras e às medidas de distanciamento social no município de Santa Cruz do Sul, Rio Grande do Sul.

\section{FUNDAMENTAÇÃO TEÓRICA}

A experiência no mundo demonstrou que incluir diversas formas de distanciamento social, desde o isolamento de casos e contatos, até o bloqueio total (lockdown), podem conter a pandemia. Porém, a aplicabilidade dessas estratégias se dá de diferentes formas entre os diversos países (SOUZA, 2020). 
Segundo Etienne (OPAS, 2020), manter medidas de distanciamento social, melhorar a vigilância e fortalecer os sistemas de saúde são as três chaves para controlar a pandemia de COVID-19 nas Américas. As Américas, uma região de enormes desigualdades, está enfrentando emergências simultâneas de saúde, econômicas e sociais durante a pandemia, que devem ser tratadas em conjunto para deter a COVID-19. Isso inclui um número acentuado de casos, economias tensas e grupos vulneráveis em maior risco, como populações indígenas, migrantes e pessoas com doenças crônicas. 0 distanciamento social diminui a transmissão para que os serviços de saúde possam testar casos suspeitos, rastrear contatos e tratar e isolar pacientes.

A situação que enfrentamos é terrível, desde que nossa abordagem para derrotar o vírus se baseie na solidariedade. Devemos trabalhar juntos, compartilhar recursos e aplicar as estratégias comprovadas que aprendemos ao longo do caminho (Etienne, 2020).

O aumento no número de casos da COVID-19 levou o governo do Brasil a medidas de saúde preventivas, como o distanciamento social e o uso de máscaras. Dentre as medidas de distanciamento social, podemos citar a paralisação de atividades não essenciais, com exceção de serviços essenciais, como supermercados e farmácias. Assim, evitando aglomerações, que propicia a disseminação do vírus (SOUZA, 2020).

Segundo Ortelan (2020), estudos anteriores à atual pandemia já haviam demonstrado a efetividade do uso de máscaras, inclusive de máscaras de tecido, em locais extradomiciliares como estratégia de controle à disseminação de doenças infecciosas respiratórias.

Com o avanço da pandemia da COVID-19, argumentações sobre o uso de máscaras em ambientes públicos, como intervenção em saúde pública, começaram a ser discutidas. Diversos fatores foram debatidos como: essa recomendação poderia diminuir as medidas de distanciamento social, a manipulação e o uso inadequados das máscaras poderia elevar o risco de transmissão do vírus, a demanda de máscaras poderia causar escasez de máscaras cirúrgicas para profissionais de saúde e essa recomendação poderia oportunizar a prática de preços abusivos (SOUZA, 2020).

As máscaras de tecido apresentam menor eficácia, pois elas funcionam como barreira mecânica à transmissão do vírus, impedindo ou reduzindo o contato dos indivíduos com gotículas contaminadas, do que comparadas às máscaras cirúrgicas. Se usadas corretamente, as máscaras podem evitar a contaminação de seus usuários, ajudando a diminuir a velocidade de evolução da doença; é válido pontuar que o uso da máscara deve ser bem orientado, já que se usada incorretamente, sua eficácia pode diminuir em até 60\% (ORTELAN, 2020).

Seguindo a linha de pensamento do autor descrito anteriormente, desde o início da pandemia muitos países, mesmo sem comprovação do uso de máscaras, decretaram que toda a população deveria utilizar o equipamento de proteção individual. Ainda que diversos estudos e governantes indiquem a eficácia e a importância do uso de máscaras no controle da pandemia, a taxa de adesão pelas populações varia notoriamente. Algumas pesquisas mostram que a efetividade do uso das máscaras de tecido, para o controle da COVID-19, é bem suportada em conjunto com outras medidas de combate à pandemia, como a higienização das mãos e o distanciamento social. Em decorrência desse fato, é válido ressaltar a importância da educação em saúde nos tempos atuais, pois o uso das máscaras de tecido, sem a adoção destas outras medidas, não poderá ser visto pelos usuários como solução para a crise atual.

Segundo Natividade (2020), o emprego e o medo do desemprego são questões a serem consideradas, pois torna a população vulnerável aos riscos do novo coronavírus. Ressalta-se ainda que pessoas com menor renda ou 
assalariados, além de mais expostas aos impactos financeiros gerados pela pandemia da COVID-19, também estão mais vulneráveis a sofrerem com problemas de saúde de âmbito físico, psíquico e social associados ao plano de contingência necessário no período de isolamento social.

Segundo Iwaya (2020) apud Van Bavel et al. (2020), buscar apoios religiosos e políticos pode ser uma estratégia eficaz na disseminação de mensagens persuasivas em campanhas de saúde pública durante o atual momento. Durante a crise de Ebola na África, por exemplo, o engajamento dos líderes religiosos em campanhas que defendem boas práticas foi considerado o ponto de virada na resposta à epidemia. As crenças exercem maior influência para que a população entenda os benefícios de permanecer em distanciamento social.

Segundo Moreira (2020), outras formas de enfrentamento aos impactos gerados pelo isolamento social e promoção de proteção à saúde mental por meio do bem-estar psicológico estão entrando em discussão. 0 presente estudo evidenciou oportunidades e desafios na utilização de aplicativos que integram atividade física a jogos digitais. Neste sentido, é importante o desenvolvimento de intervenções de cuidados em saúde mental coletivas, baseadas em evidências que considerem o contexto atual de pandemia e os fatores que desencadeiam problemas de saúde decorrentes do isolamento imposto pelas novas regras de saúde.

\section{MATERIAIS E MÉTODOS}

Este estudo partiu de um recorte de um estudo transversal de base populacional que mensurou a soroprevalência de SARS-CoV-2 na região de abrangência do Consórcio Intermunicipal de Serviços do Vale do Rio Pardo - CISVALE, que compreende 14 municípios do Vale do Rio Pardo, região central do estado do Rio Grande do Sul. As cidades participantes foram: Boqueirão do Leão, Candelária, Gramado Xavier, Herveiras, Mato Leitão, Pantano Grande, Passo do Sobrado, Rio Pardo, Santa Cruz do Sul, Sinimbu, Vale do Sol, Vale Verde, Venâncio Aires e Vera Cruz. Neste recorte são apresentados os dados referentes às três primeiras etapas, considerando apenas os dados coletados no município de Santa Cruz do Sul.

As coletas ocorreram nos finais de semana a fim de obter uma maior regularidade de indivíduos nas residências. As três primeiras etapas apresentaram intervalos de duas semanas, em que a primeira ocorreu em 1 e 2 de agosto e a última em 12 e 13 de setembro de 2020 (da $31^{\circ}$ até a $38^{\mathrm{a}}$ semana do calendário epidemiológico).

0 processo de amostragem foi realizado por múltiplos estágios, incluindo amostragem sistemática dos setores censitários, seguido de sorteio aleatório simples dos domicílios em cada setor conforme tamanho de amostra estipulado. Os endereços que foram pesquisados em cada setor sorteado foram obtidos a partir do Cadastro Nacional de Endereços para Fins Estatísticos (CNEFE) do IBGE, Censo de 2010. Em cada domicílio uma pessoa foi aleatoriamente sorteada e convidada a participar do estudo. A partir da segunda rodada, os domicílios participantes nos setores censitários urbanos foram sempre selecionados de forma sistemática, a partir de um pulo de cinco domicílios à direita a contar do domicílio selecionado na rodada anterior. Nas situações em que o domicílio selecionado não correspondia ao endereço residencial, ou que não havia ninguém no momento da entrevista ou diante de recusa em participar do estudo, o mesmo foi substituído pelo próximo domicílio à direita que atendesse aos critérios de inclusão. Para os setores censitários de zona rural, em todas as etapas foi sorteado um novo endereço como ponto de partida para as coletas. 
Em cada domicílio, após o sorteio aleatório, o morador em caso de aceite assinava os termos de consentimento e era realizado o exame de teste de diagnóstico rápido para a detecção qualitativa de anticorpos IgG e IgM contra SARS-CoV-2. Foi utilizado um teste rápido (TR) imunocromatográfico qualitativo (Leccurate do fabricante Beijing Lepu Medical Techonology CO LTD) em amostra de sangue coletada por punção digital. Ao aguardar o resultado do exame (tempo de no máximo 15 minutos), foram coletados dados para caracterização da amostra por meio de um formulário no software Epi Info ${ }^{\mathrm{TM}}$. Em caso de teste rápido reator, todos os moradores do domicílio presentes no momento da coleta de dados foram convidados a também realizar o teste rápido e a vigilância epidemiológica do município foi comunicada dos resultados.

A coleta de dados em Santa Cruz do Sul foi realizada por estudantes selecionados entre os cursos da área da saúde da UNISC. Todos os entrevistadores receberam treinamento sobre a realização do TR, normas de biossegurança, bem como aplicação do questionário antes de cada etapa.

Para a caracterização da amostra foram analisadas as variáveis sexo, faixa etária, escolaridade, renda familiar mensal (categorização em salários mínimos) e zona de moradia (urbana e rural). Como variável de desfecho neste estudo, foram avaliadas: distanciamento social através da pergunta "Com relação ao distanciamento social que está sendo orientado pelas autoridades de saúde, ou seja, ficar em casa e evitar contato com outras pessoas, quanto acha que está conseguindo fazer (muito pouco, pouco, mais ou menos, bastante praticamente isolado do mundo)" e uso de máscara através do questionamento: "Você usa máscara quando sai de casa?" (sim, não, às vezes).

A consistência e análise dos dados foram realizadas no software Stata ${ }^{\circledR} 14.0$ (StataCorp. 2013. Statistical Software: Release 14. College Station, TX: StataCorp LP) versão 11. Foi realizada análise estatística descritiva de forma que as variáveis categorias foram representadas por meio de frequência absoluta e relativa, e as variáveis numéricas por meio de média e desvio padrão

A pesquisa foi aprovada pelo Comitê de Ética em Pesquisa da UNISC sob o parecer número 4.193.725. Todos os participantes receberam informações detalhadas sobre os objetivos da pesquisa, os riscos e os benefícios de sua participação.

\section{RESULTADOS E DISCUSSÃO}

A amostra foi composta por 386 indivíduos em cada rodada, totalizando 1158 indivíduos entrevistados. A média de idade foi de 49,5 ( \pm 19 ) anos. As características predominantes dos participantes foram: sexo feminino (62,72\%), população adulta, economicamente ativa na faixa etária de 20 a 59 anos (58,7\%), ensino fundamental (40,35\%), renda familiar entre $\mathrm{R} \$ \mathbf{1 . 0 4 6 , 0 0}$ até $\mathrm{R} \$ \mathbf{3 . 1 3 5 , 0 0}(43,92 \%)$ e moradia em zona urbana $(80,40 \%)$, conforme os dados apresentados na Tabela 1. 
Tabela 1. Descrição da amostra conforme variáveis demográficas e socioeconômicas.

Total

n $\%$

Sexo

Masculino

431

37.3

Feminino

725

62.7

\section{Faixa etária}

0 a 19 anos

75

6.6

20 a 59 anos

662

58.7

$\geq 60$ anos

399

34.6

Escolaridade

Analfabeto/não estudou

18

1.6

Educação infantil

48

Fundamental

464

40.4

Ensino Médio

339

29.5

Superior e Pós-graduação

281

24.4

\section{Renda Familiar*}

Até $\mathrm{R} \$ 1045,00$

De $\mathrm{R} \$ 1046,00$ até $\mathrm{R} \$ 3135,00$

491

43.9

De R\$ 3136 até $\mathrm{R} \$ \mathbf{6 2 7 0 , 0 0}$ 
Não quis informar

\section{Zona de moradia}

Urbana

Rural
76

6.8

Foi constatado que a minoria dos entrevistados, $4,41 \%$ relatam que seguem muito pouco o distanciamento social, contudo, 55,7\% responderam que segue as medidas de distanciamento, como mostra na Figura 1.

Figura 1: Prevalência do Distanciamento Social, Santa Cruz do Sul (n=1158), 2020.

\section{DISTANCIAMENTO SOCIAL}

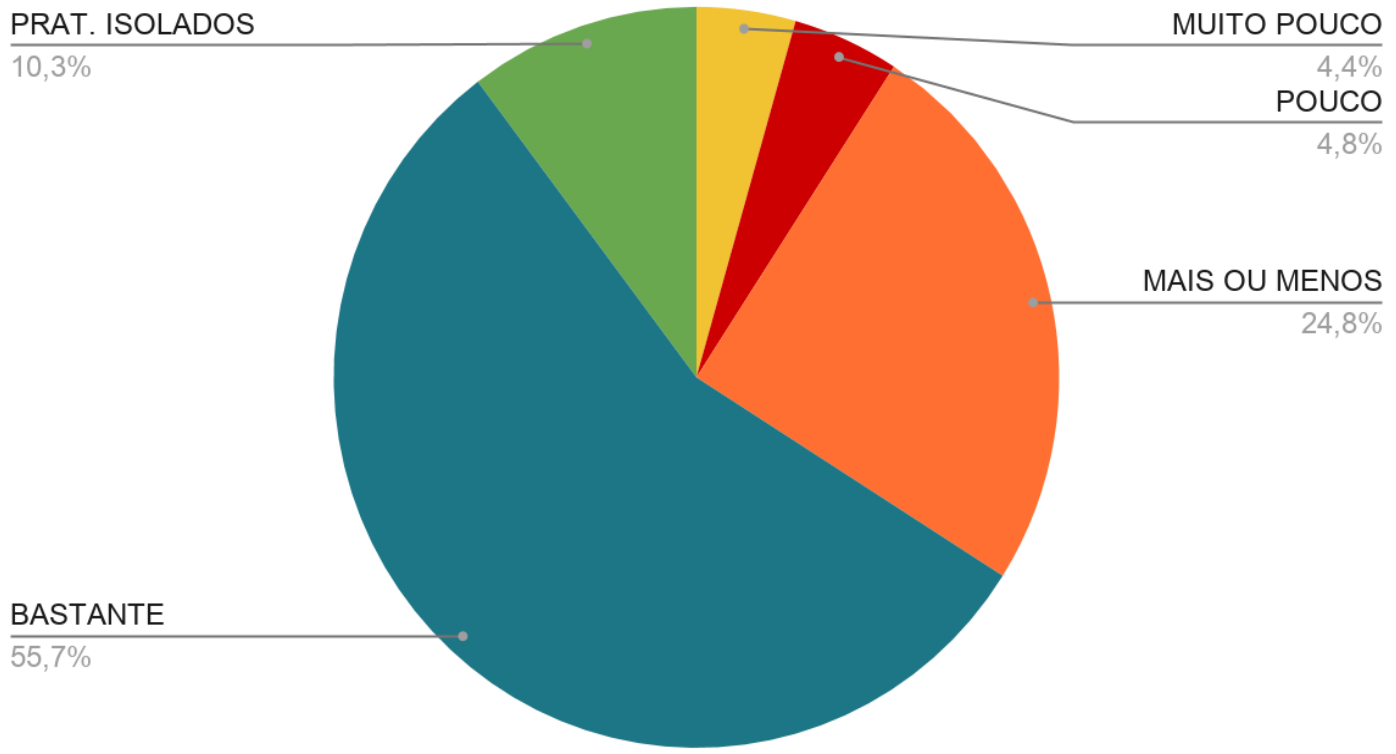

Fonte: Dados da pesquisa.

Também foram encontrados valores em relação ao uso de máscara ao sair de casa, $0,88 \%(10)$ responderam que não fazem uso de máscara ao sair de casa, 3.45\% (39) dos entrevistados responderam que usam máscara às vezes ao sair de casa e 95,66\% (1081) relatam que usam máscara sempre ao sair, como mostra a Figura 2. 
Figura 2: Prevalência do uso de máscara ao sair de casa, Santa Cruz do Sul (n=1158), 2020.

\section{USO DE MÁSCARA AO SAIR DE CASA}

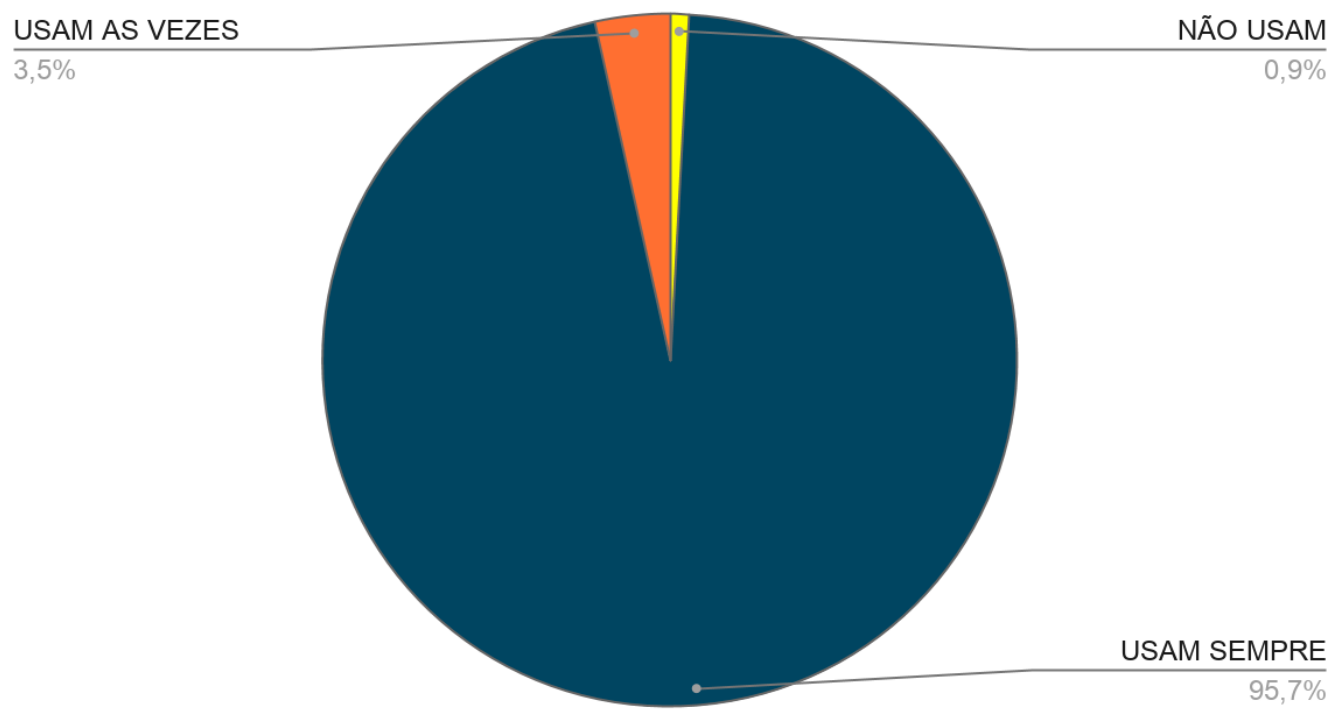

Fonte: Dados da pesquisa.

0 resultado da pesquisa mostra-se positivo, sendo que os indicadores propostos evidenciam que $50 \%$ da população está conseguindo manter o distanciamento social corretamente, e agregado ao uso contínuo de máscara, mais de $90 \%$ em lugares públicos.

Segundo Wilder et al. (2020), essas medidas de saúde pública são ferramentas que possuem um efeito positivo para interromper a transmissão dessa doença contagiosa, mas que não deixam de ser um grande desafio para a sociedade. 0 mesmo completa que o objetivo principal da implementação do distanciamento social é o controle de doenças transmissíveis por meio da redução de interação entre a população da comunidade, pelo fato de que alguns indivíduos da sociedade se encontram infectados, mas não identificados e, consequentemente, não isolados, causando a propagação da doença por meio de suas gotículas respiratórias.

Segundo o Centers for Disease Control and Prevention (CDC, 2021), reduzindo a transmissão entre grupos, diminui-se o número de atendimentos urgentes, não ocasionando a superlotação do sistema de saúde que demandaria grande número de profissionais, leitos e aparelhos de saúde necessários para tais cuidados. Dessa forma, evita-se o colapso, caos e mortes desnecessárias, conseguindo atingir o objetivo: achatar a curva epidemiológica. Além disso, existem grandes desafios para a implementação das medidas restritivas, em que a cooperação coletiva da comunidade pode ter uma grande resistência por enxergarem tais medidas como contenção da liberdade (cancelar ou substituir atividades), o que requer uma ótima comunicação com a população para explicar e justificar cada ação e sua importância para o atual momento de crise de saúde pública. Todavia, a única certeza que se tem é de que as decisões futuras sobre o momento e a duração da flexibilização de modo seguro precisarão ser informadas por uma vigilância epidemiológica contínua e rigorosa. Ao aderir às novas normas de contingência, as pessoas se sentem isoladas de amigos e familiares, além de também estarem sujeitas a uma possibilidade de desemprego, o que dificulta aceitação e execução dessa solicitação que carece de apoio emocional e financeiro. 
Segundo Camargo et al. (2020), tendo em vista a gravidade da situação, o uso de EPI se transformou em uma premência não somente para doentes e profissionais da área da saúde, mas sim para toda a população por ser uma contenção da propagação da doença. Os autores também destacam a eficácia do uso de máscaras para o enfrentamento da pandemia atual. São recomendadas máscaras cirúrgicas e de proteção de uso individual que filtram aerossóis e protegem de agentes infecciosos, sendo que a máscara de tecido não é recomendada para ambientes odonto-médico-hospitalares por possuir uma limitação na sua filtração nesses locais específicos, mas é comprovado que ela tem sido uma aliada na barreira contra gotículas frente à pandemia.

Em síntese, consegue-se afirmar que, segundo Ortelan et al. (2021), as máscaras de tecido em ambiente extradomiciliar têm sido positivas para o controle de transmissão do SAR-CoV-2. Com evidências acumuladas nacional e internacionalmente, é notório que além de reduzir o risco de propagação do vírus, evita-se o descarte inapropriado de máscaras descartáveis e com elas um foco de infecção, assim como a redução da carga de doenças da população por outras doenças infectocontagiosas. As máscaras de tecido são uma intervenção de saúde pública que, vinculada com distanciamento social e higienização das mãos, é possível reduzir a disseminação de COVID-19. Esse simples esforço é capaz de se tornar um grande ato ao reduzir o número de casos e óbitos.

\section{CONCLUSÃO}

É notório que a implementação do distanciamento social e o uso de máscaras é um grande desafio na realidade do nosso país. Apesar disso, verificou-se que mais da metade da população entrevistada segue as medidas de mitigação da pandemia de COVID-19. Essa estratégia implementada é de extrema importância para a não sobrecarregar os serviços de saúde e redução do número de mortes, de forma que uma adesão efetiva garante a proteção das comunidades da região.

\section{AGRADECIMENTOS}

Os autores agradecem ao CISVALE (Consórcio Intermunicipal de Serviços do Vale do Rio Pardo), a 13 ${ }^{\mathrm{a}}$ Coordenadoria Regional de Saúde do Rio Grande do Sul, as Prefeituras Municipais de Boqueirão do Leão, Candelária, Gramado Xavier, Herveiras, Mato Leitão, Pantano Grande, Passo do Sobrado, Rio Pardo, Santa Cruz do Sul, Sinimbu, Vale do Sol, Vale Verde, Venâncio Aires e Vera Cruz, e à Philip Morris Brasil, pelo apoio ao desenvolvimento desta pesquisa.

\section{REFERÊNCIAS}

ORTELAN, Naiá et al. Máscaras de tecido em locais públicos: intervenção essencial na prevenção da COVID-19 no Brasil. Ciência \& Saúde Coletiva, v. 26, p. 669-692, 2021. Disponível em:

<https://www.scielosp.org/article/csc/2021.v26n2/669-692/> Acesso em 27 de março de 2021.

AQUINO, Estela ML et al. Medidas de distanciamento social no controle da pandemia de COVID-19: potenciais impactos e desafios no Brasil. Ciência \& Saúde Coletiva, v. 25, p. 2423-2446, 2020. Disponível em:< https://www.scielosp.org/article/csc/2020.v25suppl1/2423-2446/pt/\#> Acesso em 26 de março de 2021. 
Brasil. Ministério da Saúde (MS). Sobre a doença. Brasilia: MS; 2020. Disponível em: <https://coronavirus.saude.gov.br/sobre-a-doenca> Acesso em 26 de março de 2021.

MEMON, Zaibunnisa; QURESHI, Sania; MEMON, Bisharat Rasool. Assessing the role of quarantine and isolation as control strategies for COVID-19 outbreak: a case study. Chaos, Solitons \& Fractals, v. 144, p. $110655,2021$. Disponível em:<https://www.sciencedirect.com/science/article/pii/S0960077921000084\#sec0001> Acesso em 27 de março de 2021.

MOREIRA, Wanderson; SOUSA, kayo; SOUSA, Anderson. Intervenções em saúde mental em tempos de COVID19: scoping review. Disponível em: <file:///C:/Users/Gabriela/Downloads/1007-Preprint\%20Text-1521-2-1020200723.pdf> Acesso em 2 de maio de 2021.

Wilder-Smith A, Freedman DO. Isolation, quarantine, social distancing and community containment: pivotal role for old-style public health measures in the novel coronavirus (2019-nCoV) outbreak. J Travel Med 2020; 27:2. Disponível em:<https://pubmed.ncbi.nlm.nih.gov/32052841/> Acesso em 26 de março de 2021.

OPAS. Distanciamento social, vigilância e sistemas de saúde mais fortes são chaves para controlar a pandemia de COVID-19, afirma diretora da OPAS. Disponível em:<https://www.paho.org/pt/noticias/2-6-2020-

distanciamento-social-vigilancia-e-sistemas-saude-mais-fortes-sao-chaves-para>Acesso em: 06 de abril de 2021.

CONASS. Painel CONASS Covid-19. Disponível em:<https://www.conass.org.br/painelconasscovid19/>Acesso em: 25 de março de 2021.

SOUZA, Mariluce Karla Bomfim. Medidas de distanciamento social e demandas para reorganização dos serviços hemoterápicos no contexto da Covid-19. Disponível

em:<https://www.scielo.br/scielo.php?script=sci_arttext\&pid=S1413-81232020001204969\&lang=pt $>$ Acesso em 29 de Março de 2021.

ORTELAN, Naiá; FERREIRA, Andrêa; LEITE, Luciana; et al. Máscaras de tecido em locais públicos: intervenção essencial na prevenção da COVID-19 no Brasil. Disponível

em:<https://www.scielo.br/scielo.php?script=sci_arttext\&pid=S1413-81232021000200669\&lang=pt $>$ Acesso em 29 de março de 2021.

Interventions for Community Containment. Disponível em:<https://www.cdc.gov/sars/guidance/dquarantine/app1.html> Acesso em 27 de março de 2021.

NATIVIDADE, Márcio; BERNARDES, Kionna; PEREIRA, Marcos; et al. Distanciamento social e condições de vida na pandemia COVID-19 em Salvador-Bahia, Brasil. Disponível

em:<https://www.scielo.br/scielo.php?script=sci_arttext\&pid=S1413-81232020000903385\&lang=pt $>$ Acesso em 29 de Março de 2021.

IWAYA, Gabriel; CARDOSO, Janaína; JÚNIOR, João; et al. Preditores da intenção de permanecer em distanciamento social. Disponível em:<https://www.scielo.br/scielo.php?script=sci_arttext\&pid=S0034$76122020000400714 \&$ lang=pt> Acesso em 30 de Março de 2021.

CAMARGO, Maria Cristina de et al. Eficácia da máscara facial (TNT) na população para a prevenção de infecções por coronavírus: revisão sistemática. Ciência \& Saúde Coletiva, v. 25, p. 3365-3376, 2020. Disponível em: <https://doi.org/10.1590/1413-81232020259.13622020> Acesso em 28 de março de 2021. 